$$
\begin{array}{lll} 
& \text { W. K. H. Panofsky } \\
\text { JAN } 101991 & \text { November 21, } 1990
\end{array}
$$

\title{
SPIN MOTION OF ELECTRONS IN THE SLC LINAC
}

It is generally expected that the depolarizing effects of the linear accelerator R.F. fields will be small. Recently Bill Atwood ${ }^{\star}$ raised the question whether this conclusion is still correct in view of the fact that the particles in the SLC spend a larger fraction of their time at phase angles "off crest" due to BNS damping; since radial fields are in quadrature with the accelerating field this might imply that depolarizing effects are larger. On the other hand, because of the smaller emittance of the SLC relative to the earlier linac radial excursions would be smaller. The anticipation is therefore that the depolarizing effect will again be negligible but it might be worthwhile to update the early calculations of SLAC TN-63-97 revised. That earlier TN had minor errors which were pointed out by Ed McMillan and others.

In the rest frame of the electron the spin will rotate at an angular velocity $\Omega_{R}$ given by

$$
\vec{\Omega}_{R}=\frac{g e}{2 m_{o}} \vec{B}_{o}
$$

where the magnetic field $\vec{B}_{0}$ in the rest frame is related to the fields in the lab by

$$
\vec{B}_{0}=\gamma\left[\vec{B}-\left(\frac{\gamma}{\gamma+1}\right)(\vec{v} \cdot \vec{B}) \vec{v}-\vec{v} \times \vec{E}\right]
$$

In the paraxial approximation the radial electric field $E_{r}$ and azimuthal magnetic

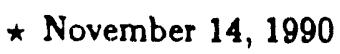

Work supported by the Department of Energy Contract DE-AC03-76SF00515.

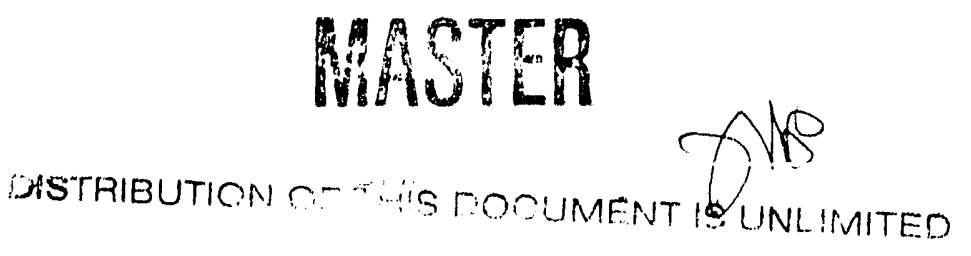


field $B_{\phi}$ are related to the accelerating field $E_{z}$ by

$$
E_{r}=\frac{-r}{2} \frac{\partial E_{z}}{\partial z} ; B_{\phi}=\frac{r}{2} \frac{\partial E_{z}}{\partial t}
$$

- If there are no orbits of angular momentum about the $z$ axis, then $\vec{v} \cdot \vec{B}=0$ and $\vec{B}_{o}$ in the rest frame has only an azimuthal component. Therefore $B_{0}$ is given by

$$
B_{0}=\gamma\left(B_{\phi}-v E_{\mathrm{r}}\right)+p_{\mathrm{r}} E_{z} / m_{0}
$$

where $p_{r}=\gamma m_{0} v_{r}$ is the radial momentum; let us from now on take $m_{0}=c=1$, i.e. electric fields are measured in units of $m_{0} c^{2}$ per unit length.

The precession rate $\Omega_{L}$ in the laboratory is given by

$$
\Omega_{L}=\frac{1}{\gamma} \Omega_{R}+\omega_{T}
$$

where

$$
\omega_{T}=-(\gamma-1) \omega
$$

is the Thomas precession ${ }^{\dagger}$ which is a purely kinematic relativistic effect; here $\omega$ is the laboratory rotation rate of the electron orbit given by

$$
-\omega=\frac{1}{v^{2}}\left|\vec{v} \times \frac{d \vec{v}}{d t}\right|
$$

Since

$$
\frac{d \vec{v}}{d t}=\frac{d}{d t}\left(\frac{\vec{p}}{\gamma}\right)=\frac{1}{\gamma} \frac{d \vec{p}}{d t}+\vec{p} \frac{d\left(\frac{1}{\gamma}\right)}{d t}
$$

we have

$$
-\omega=\frac{1}{p_{z}} \frac{d p_{r}}{d t}-\frac{p_{r}}{p_{z}^{2}} \frac{d p_{z}}{d t}
$$

† See e.g. Møller, The Theory of Relativity, Oxford 1952, pp $53 \mathrm{ff}$. 
From (3):

$$
\frac{d p_{r}}{d t}=-\frac{r}{2}\left(\frac{\partial E_{z}}{\partial z}+v \frac{\partial E z}{\partial t}\right)=-\frac{r}{2}\left(\frac{1}{\gamma^{2}} \frac{\partial E_{z}}{\partial z}+v \frac{d E_{z}}{d t}\right)
$$

where

$$
\frac{d}{d t}=\frac{\partial}{\partial t}+v \frac{\partial}{\partial z}
$$

is the total time derivative referred to the moving charge and $E_{z}=d p_{z} / d t$. The total laboratory precession rate given by (5) then becomes

$$
\begin{aligned}
\Omega_{2} & =\frac{g}{2 \gamma}\left[\frac{r \gamma}{2}\left(\frac{\partial E_{z}}{\partial t}+v \frac{\partial E_{z}}{\partial z}\right)+p_{r} E_{z}\right] \\
& -\frac{(\gamma-1) r}{2 v \gamma}\left[\frac{\partial E_{z}}{\partial z}+v \frac{\partial E_{z}}{\partial t}\right]-\frac{(\gamma-1)}{\gamma^{2} v^{2}} p_{r} E_{z} \\
& =a\left[\frac{r}{2} \frac{d E_{z}}{d t}+\frac{p_{r} E_{z}}{\gamma}\right]+\frac{1}{\gamma} \frac{r}{2} \frac{d E_{z}}{d t}-\left(1-\frac{1}{\gamma}\right) \frac{1}{v \gamma^{2}} \frac{r}{2} \frac{\partial E_{z}}{\partial z}+\frac{p_{r} E_{z}}{\gamma(\gamma+1)}
\end{aligned}
$$

where $a=(g-2) / 2=1.2 \times 10^{-3}$ is the gyromagnetic anomaly. To obtain the total precession angle eq. (11) must be integrated over time. We can simplify the integration by noting that

$$
r \frac{d E_{z}}{d t}=\frac{d}{d t}\left(r E_{z}\right)-\frac{E_{z} p_{r}}{\gamma}
$$

and observing that the total derivative in the first term of the right hand side of eq. (12) will not contribute to the time integral over the precession rate. Therefore after some simplification the precession angle $\Theta_{L}$ is given by

$$
\Theta_{L}=\int\left\{\frac{a}{2} \frac{p_{r} E_{z}}{\gamma}+\frac{p_{r} E_{z}}{2 \gamma^{2}}\left(\frac{\gamma-1}{\gamma+1}\right)-\frac{r}{2 \gamma^{2}}\left(\frac{\gamma-1}{\gamma+1}\right)^{1 / 2} \frac{\partial E_{z}}{\partial z}\right\} d t
$$

Let us write

$$
d t=d \gamma / E_{z} v
$$


Then $\Theta_{L}$ becomes

$$
\Theta_{L}=\int_{1}^{\gamma_{\max }}\left\{\frac{a}{2} \frac{p_{r}}{\sqrt{\gamma^{2}-1}}+\frac{p_{r}}{2 \gamma} \frac{(\gamma-1)^{1 / 2}}{(\gamma+1)^{3 / 2}}-\frac{r}{2 \gamma}\left(\frac{1}{\gamma+1}\right) k \tan \alpha\right\} d \gamma
$$

where

$$
\frac{1}{E_{z}} \frac{\partial E_{z}}{\partial z} \sim k \tan \alpha
$$

where $k=2 \pi / \lambda$ is the R.F. inverse wavelength and $\alpha$ is the off-crest phase angle of the particle.

The quantities $r$ and $p_{r}$ are oscillating corresponding to the invariant emittance $\epsilon_{n}$ and focusing parameter $\beta$; Their amplitudes are

$$
p_{r} \sim \gamma \sqrt{\left(\epsilon_{n} / \beta \gamma\right)} \quad r \sim \sqrt{\epsilon_{n} \beta / \gamma}
$$

Let us take

$$
\begin{aligned}
\epsilon_{n} & \sim 3 \times 10^{-5} \text { radian meter } \\
\tan \alpha & \sim 0.2\left(11^{\circ}\right) \\
k & =20 \pi \text { meter }^{-1} \\
a & =1.2 \times 10^{-3} .
\end{aligned}
$$

Let us take $r=10^{-3} \mathrm{~m}$ at the front end. Then roughly $p_{r} \cong 10^{-2} \gamma^{1 / 2}$, $r \cong 10^{-3} \gamma^{-1 / 2}$, and $k r \tan \alpha=0.06 \gamma^{-1 / 2}$.

The integration should be extended to a $\gamma$ value corresponding to a few betatron wavelengths but in practice we can take $\gamma_{\max }=\infty$. The first term is negligible due to the small value of $\mathrm{g}-2$; the second term is of order $10^{-3}$. The integral in the last term is

$$
\int_{1}^{\infty} \frac{d \gamma}{\gamma^{3 / 2}(\gamma+1)}=1-\pi / 4
$$

Thus under these conservative assumptions the last term is about 0.012 . Thus indeed, as suggested the large phase angle corresponding to BNS damping makes 
the last term dominant but still acceptably small. Note that the phase angle estimate is very conservative since only its value early in the machine contributes.

In summary, there should be no depolarization problem stemming from the R.F. fields of the linac, but a numerical integration of eq. (14), using actual

- SLC parameters might be indicated. Also the effect of inisalignments should be isvestigated.

\section{DISCLAIMER}

This report was prepared as an account of work sponsored by an agency of the United States Government. Neither the United States Government nor any agency thereof, nor any of their employees, makes any warranty, express or implied, or assumes any legal liability or responsibility for the accuracy, completeness, or usefulness of any information, apparatus, product, or process disclosed, or represents that its use would not infringe privately owned rights. Referproce herein to any specific commercial product, process, or service by trade name, trademark, manufacturer, or otherwise does not necessarily constitute or imply its endorsement, recomme United States Government or any agency thereof. The views and opinions of authors expressed herein do not necessarily state or reflect those of the United States Government or any agency thereof. 

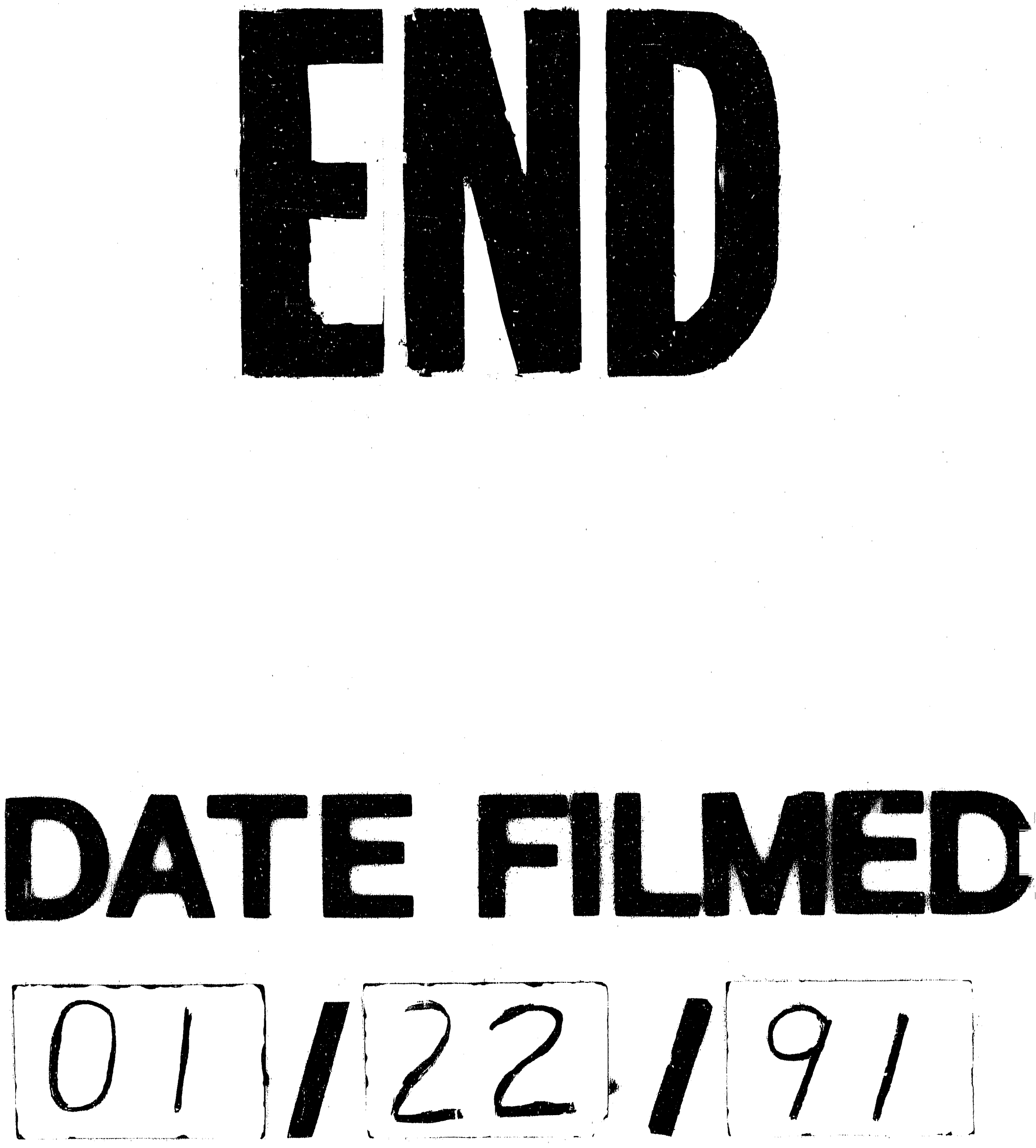
